\title{
A telehealth approach to improving clinical trial access for infants with tuberous sclerosis complex
}

Carly Hyde ${ }^{1^{*}} \mathbb{D}$, Maria Pizzano ${ }^{2}$, Nicole M. McDonald ${ }^{3}$, Charles A. Nelson $1 I^{4}$, Connie Kasari ${ }^{3}$, Elizabeth A. Thiele ${ }^{5}$ and Shafali S. Jeste ${ }^{3}$

\begin{abstract}
Background: Research in rare genetic syndromes associated with ASD is often hampered by the wide geographic distribution of families and the presence of medical comorbidities, such as epilepsy, that may preclude travel to clinical sites. These challenges can limit the sample size and generalizability of the cohorts included in both natural history studies and clinical trials. Tuberous sclerosis complex (TSC) is a rare genetic syndrome that confers an elevated risk for autism spectrum disorder (ASD), with social communication delays identified in this population as early as 12 months of age. Early identification of risk necessitates parallel testing of early intervention, prompting the first randomized controlled clinical trial of behavioral intervention for infants with TSC (NCT03422367). However, considerable early recruitment challenges have mandated the systematic identification of enrollment barriers followed by modification of the study design to address these barriers.
\end{abstract}

Methods: Caregivers were interviewed regarding barriers to enrollment (phase 1). Adaptations to the intervention were made to address these barriers (phase 2). Outcomes based on this modification to the study design were defined by enrollment rate and participant demographics.

Results: Qualitative reports from caregivers indicated that distance and time were the primary barriers to clinical trial enrollment. The intervention was then modified to a remote model, with at-home, parent-delivered intervention, and weekly video conferencing with interventionists at the study sites. Enrollment increased 10-fold (from 3 to 30 participants) within 1 year and included a more diverse and clinically representative cohort of infants.

Conclusion: The design and implementation of more scalable methods to disseminate research remotely can substantially improve access to clinical trials in rare neurodevelopmental disorders. The lessons learned from this trial can serve as a model for future studies not only in rare conditions, but in other populations that lack adequate access, such as families with limited financial or clinical resources. Continued efforts will further refine delivery methods to enhance efficiency and ease of these delivery systems for families.

Keywords: Telehealth, Early intervention, Tuberous sclerosis complex, Clinical trial recruitment, Behavioral intervention, Autism spectrum disorder, Remote delivery

\footnotetext{
* Correspondence: chyde@mednet.ucla.edu

'UCLA Semel Institute for Neuroscience and Human Behavior, Los Angeles,

CA 90095, USA

Full list of author information is available at the end of the article
}

(c) The Author(s). 2020 Open Access This article is distributed under the terms of the Creative Commons Attribution 4.0 International License (http://creativecommons.org/licenses/by/4.0/), which permits unrestricted use, distribution, and reproduction in any medium, provided you give appropriate credit to the original author(s) and the source, provide a link to the Creative Commons license, and indicate if changes were made. The Creative Commons Public Domain Dedication waiver (http://creativecommons.org/publicdomain/zero/1.0/) applies to the data made available in this article, unless otherwise stated. 


\section{Background}

Over the last decade, a host of identified genetic etiologies associated with autism spectrum disorder (ASD) have helped to disentangle the vast heterogeneity of the condition and inform more targeted treatments. Each of these genetic syndromes is rare (with prevalence rates ranging from $1 / 50,000$ to $1 / 6000$ ), but together they account for up to $15 \%$ of ASD [1]. Children with "syndromic" autism share common clinical features, including varying degrees of co-occurring conditions such as intellectual disability, motor impairment, epilepsy, sleep problems, and gastrointestinal dysfunction, necessitating timely treatments. Many of these syndromes manifest in infancy, with epilepsy or other medical comorbidities emerging well before an ASD diagnosis can be made. This early identification opens the door for preemptive interventions that might change developmental trajectories and improve outcomes. However, studies are challenged by the wide geographic distribution of families and the presence of medical comorbidities that may preclude travel to clinical sites. These challenges can limit the sample size and generalizability of the cohorts included in both natural history studies and clinical trials [2, 3]. More scalable methods to disseminate research remotely to individuals with rare disorders are urgently needed. Once developed, these methods can translate to other populations that lack adequate access, such as families with limited financial or clinical resources [4-6].

Here, we describe our experience with barriers to access during an ongoing clinical trial for behavioral intervention in infants with Tuberous Sclerosis Complex and the subsequent strategies formulated to mitigate the impact of these barriers. Tuberous sclerosis complex (TSC) is a rare autosomal dominant disorder caused by mutations in the TSC1 or TSC2 gene, occurring in 1 in 7000-13,000 children [7]. TSC is highly penetrant for ASD, with diagnostic rates approaching 60\% (compared to up to $2 \%$ in the general population) [8-10]. Moreover, because TSC is often diagnosed in utero [11, 12], these infants can be monitored for signs of atypical development well before a clinical ASD diagnosis is made. In a previous longitudinal study of early development, we found that infants with TSC who developed ASD demonstrated marked social communication and nonverbal cognitive delays by age 12 months $[13,14]$, with deficits in nonverbal communication skills such as eye contact, coordination of gaze, engagement, and social referencing. Despite these clear behavioral markers of atypical development in early infancy, most infants in this study were not receiving targeted social communication interventions, with therapeutic effort focused instead on global development (such as physical therapy to address delayed motor skills).
These natural history study findings prompted the design of a clinical trial of early behavioral intervention targeting social communication skills, with the ultimate goal of improving developmental outcomes. To target the specific nonverbal communicative delays identified in our longitudinal study, a behavioral intervention known as JASPER (Joint Attention, Symbolic Play, Engagement and Regulation) was selected. JASPER has been rigorously studied through clinical trials and has been shown to improve social communication and language skills in toddlers showing red flags for ASD [15]. The clinical trial, called JETS (JASPER Early Intervention in Tuberous Sclerosis, NCT03422367), fills a critical gap in treatment studies in TSC and is the first randomized clinical trial of early behavioral intervention for this syndrome.

There were high expectations that the target enrollment of 60 infants across two study sites would be readily achieved. The initial study included a waitlist-control design (see Fig. 1), with 12 weekly in-person visits over a 3month active intervention period to one of the two study sites (Los Angeles and Boston) for parent-education based behavioral intervention. Comprehensive in-person assessments were to be performed across four additional time points: pre-intervention, post-intervention, 6-month follow-up, and a 1-year follow-up. However, after one full year of the study, despite active national recruitment efforts supported by the TSC patient alliance group (Tuberous Sclerosis Alliance) and local TSC clinics, only 3 infants were enrolled.

In response to this recruitment challenge, a two-phase process was undertaken to (1) identify the barriers faced by interested families who decided not to enroll and then to (2) make modifications to the study design based upon these findings. Data from parent interviews on barriers to enrollment (phase 1) informed changes made to the research design to improve access (phase 2). Changes in enrollment rate and parent perception following the study design modifications motivate a discussion about the implications of this type of remote delivery for improved access in clinical trials across neurodevelopmental disorders.

\section{Phase 1: barrier identification Methods \\ Recruitment}

Recruitment of infants with TSC between 12 and 36 months for the clinical trial was attempted through a variety of well-established mechanisms: TSC specialty clinics, National TS Alliance referrals, online social media postings, and institution-specific medical record queries. Eligible infants had a clinical diagnosis of TSC, with the only exclusion being a planned epilepsy surgery during the trial period. 


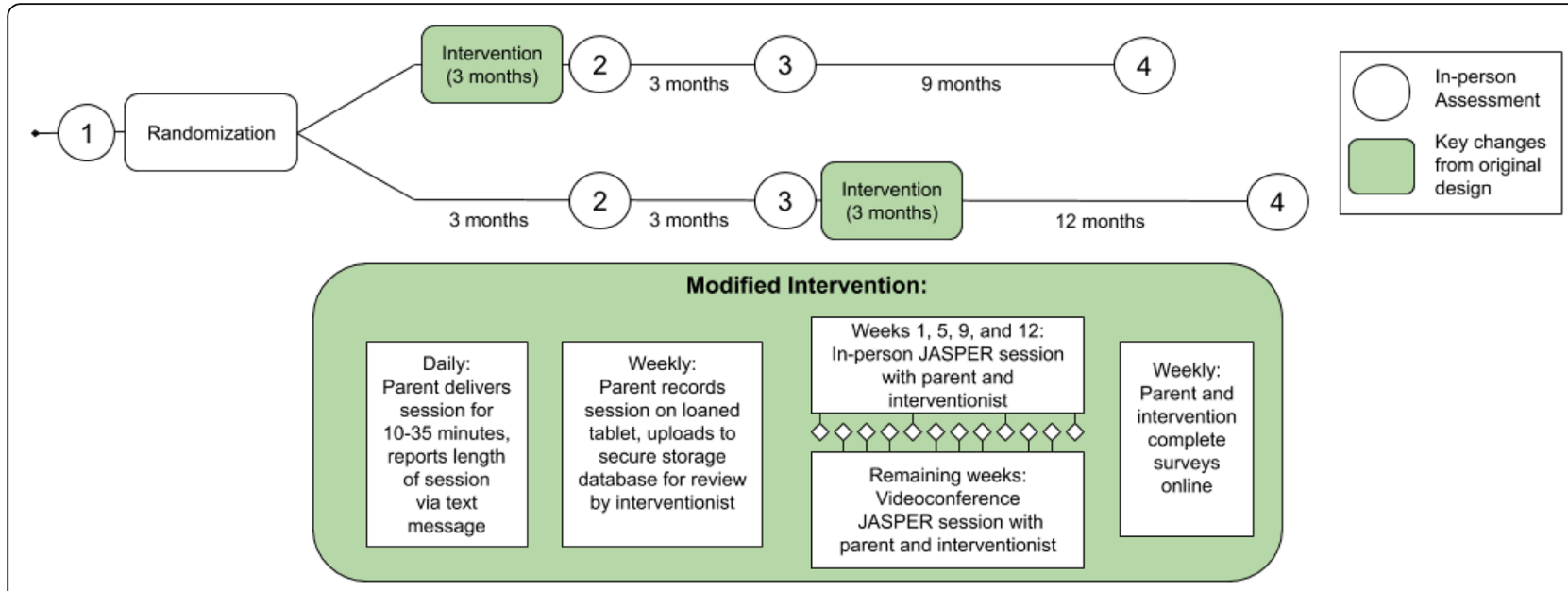

Fig. 1 Modified intervention design

\section{Screening interview}

All caregivers who responded to an advertisement or physician/alliance referral $(n=25)$ were contacted via phone. To screen infants for eligibility, caregivers were asked about the age of the child, their clinical diagnosis of TSC, and any upcoming epilepsy surgeries. All 25 of the contacted participants screened as eligible. Staff explained study details and answered questions about participation. Caregivers were then asked whether they would like to enroll in the study, either immediately following screening or after several days if parents requested time to consider.

\section{Barriers to enrollment}

Twenty-two of 25 caregivers chose not to enroll following the informational screening. These 22 families were asked two open-ended questions regarding their decision: (1) "What were the factors that led to your decision to not enroll in the study?" (2) "Are you interested in future contact if other enrollment options become available?" The results from these interviews were transcribed and categorized.

\section{Collection of demographics}

Caregivers who enrolled in the study completed a demographics questionnaire at entry, providing information about income, race and ethnicity, and education level.

\section{Results}

\section{Barrier identification}

The enrollment yield for the in-person trial design was $12 \%$. Interviews with parents who did not enroll yielded responses that fell into one of two categories, both indicating logistical challenges. The first barrier was time (27\%), which included responses related to work schedules, availability of a secondary caregiver, and frequency of appointments; the second barrier was distance (72\%) which included responses related to concern for travel costs and flying with their child (see Fig. 2). None of the responses indicated a lack of study buy-in or perceived importance of the research. All families agreed to be recontacted and many expressed a desire to participate under alternate circumstances. For example, one caregiver stated "We are extremely disappointed and hope that another trial will come up for my little one," and another commented, "If virtual ever becomes an option, we'd be interested."

\section{Phase 2: addressing barriers Methods}

Overview of remote caregiver training model

After identifying consistent challenges in recruiting families for participation in weekly in-person intervention sessions, a remote delivery method of intervention was developed to decrease demands of time and travel. The new model (Fig. 1) represents an adaptation of an existing protocol being used by Kasari et al. to remotely train JASPER interventionists nationally and internationally, with the modifications made to train caregivers [15-18]. The protocol includes teaching intervention skills and presenting curriculum content by video conference, asking trainees to practice their newly acquired techniques and to record one of the practice sessions, and submitting videos for review by a training team at one of the study sites [18]. All weekly questionnaires were digitalized. To accommodate this model for caregiver training, the study team consulted with the Security Compliance Office to identify secure and HIPAA-compliant platforms for intervention delivery, resulting in an Institutional Review Board (IRB) approved protocol. The original study design required weekly travel for $100 \%$ of sessions, while in the modified design, $70 \%$ of sessions 


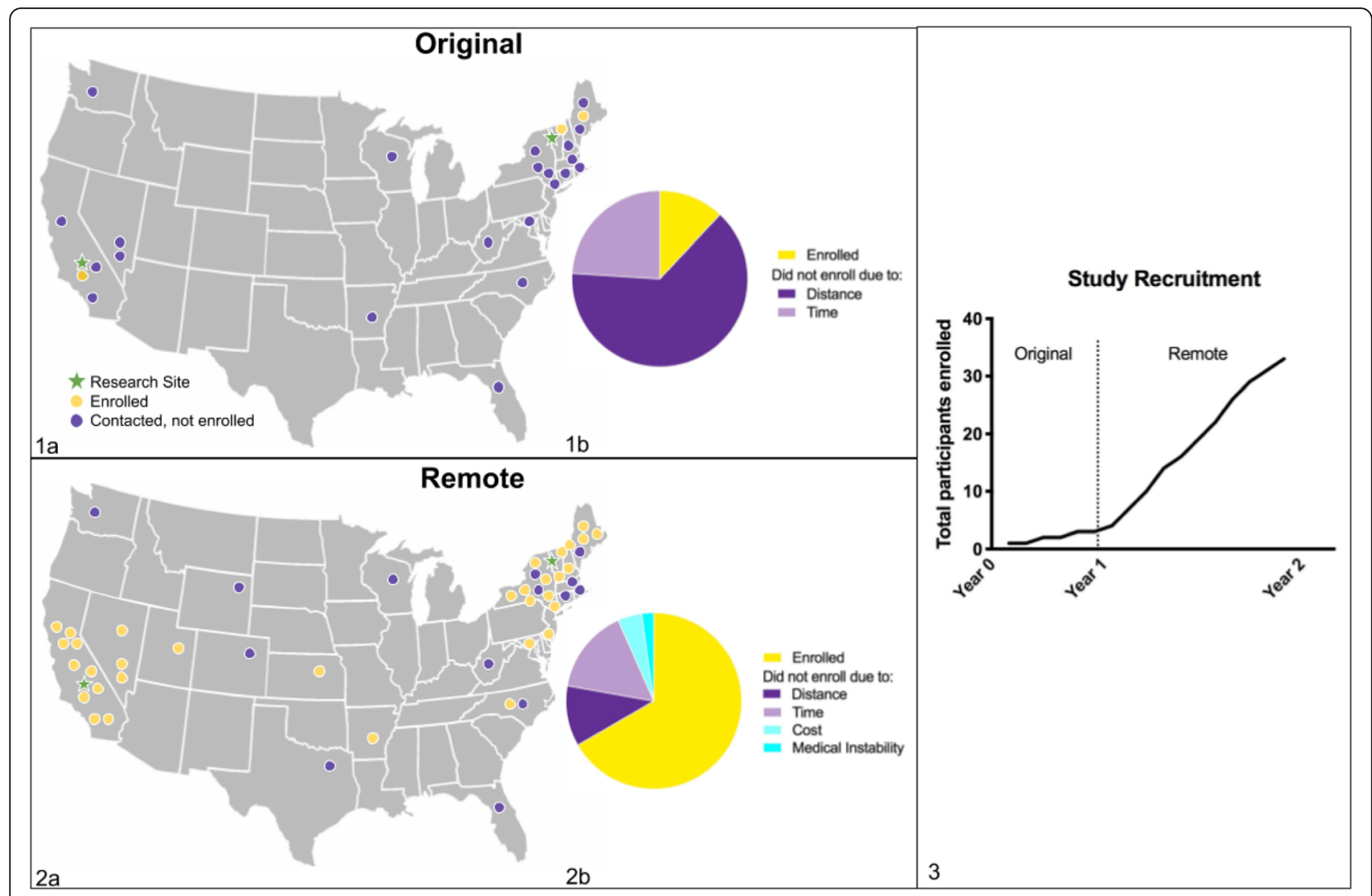

Fig. 2 Map of participants with enrollment status and breakdown of enrollment barriers for original design (1a and 1b) and remote design (2a and 2b); Total number of participants enrolled in study with original and remote design (3)

are completed remotely. This change reduced the number of in-person hours (assessments, live intervention sessions, and questionnaires) from 33 to 19 and the total number of participation hours (including video conference sessions and online questionnaires) from 39 to 30. Financial reimbursement was moderately increased from $\$ 40$ to $\$ 140$, with one night of lodging provided to families at a hotel near the study sites. Families were referred to private funding sources such as National Organization for Rare Disorders (NORD) for further reimbursement.

\section{Detailed protocol of caregiver training model}

Under the new model, caregivers first attended an inclinic intervention session focused on active coaching, live demonstration, and direct feedback. Caregivers then received an encrypted tablet to use for the duration of the intervention, with detailed operation instructions and a live demonstration. Caregivers returned home with the goal of practicing JASPER strategies daily. An automated text messaging tool allowed caregivers to easily report their intervention practice time on a daily basis by responding to nightly text. Once per week, caregivers recorded a 30-min practice video on the tablet and uploaded it to a private and secure server. After the weekly upload, a trained interventionist at one of the study sites reviewed the video. Then, during a 30-60 min weekly video conference with the caregiver, the JASPER interventionist provided feedback and introduced new JASPER content for the following week. Content built weekly to introduce new strategies and increase complexity (based on intervention presented in [16]). Weeks 1, 5, 9, and 12 (4 total) took place in-clinic; weeks $2,3,4,6,7,8,10$, and 11 (8 total) took place remotely. To collect data on parent fidelity and perspectives, caregivers and interventionists completed weekly questionnaires online through an internal institutional database. Two of these sessions ( 1 and 12) were combined with visits to study sites for in-person assessments to further reduce travel and scheduling burden on families.

Following these modifications, all 22 previously interested families were recontacted with the option to enroll in the remote intervention model. New families were also contacted for enrollment. Caregivers who chose not to enroll were administered the qualitative interview regarding their decision. 


\section{Results}

Six months after the modification to remote delivery, half of the recontacted participants $(11 / 22)$ enrolled in the study, with the remaining caregivers continuing to cite distance $(45 \%)$ or time $(54 \%)$ as barriers to enrollment. Within 1 year of the intervention modification, 23 new families expressed interest, resulting in the enrollment yield increasing to $82 \%$ for newly contacted participants (19/23). The four new caregivers who expressed interest in the study but were ultimately unable to enroll identified their primary barrier for enrollment as either time (25\%), distance (50\%), or medical instability due to epilepsy (25\%) as the primary barrier.

Although the sample size of the initial cohort was very small $(n=3)$ compared to the expanded cohort $(n=30)$, a comparison of the two cohorts for descriptive purposes was performed, indicating that the new cohort included a more diverse and representative sample of participants. The percentage of families earning $<\$ 90,000$ per year increased from 0 to $23 \%$, the percentage of families identified as non-white increased from 0 to $33 \%$, and the percentage of parents without a college degree increased from 0 to $27 \%$. Additionally, the average distance of the participants from the research site increased from 42 to 320 miles, demonstrating the improved geographical reach attained by the modified remote intervention. Following enrollment in the study, a caregiver stated "We live in a rural area where no one has heard of TSC, so it is great to be able to talk to the experts and know they have an eye on my child! I love being able to learn and add to my parent toolbox from my own home, and know I can practice these skills with her every day," while another said, "We decided when we got the diagnosis that we would do anything and go anywhere to help our son, but doing the intervention at home has been made our participation so much more manageable. I tell every parent I meet that this is a must-do."

\section{Discussion}

Here, we describe a rapid acceleration in clinical trial enrollment accomplished by the deployment of a remote intervention and assessment strategy. This modification of trial design was necessitated by barriers to access that precluded initial patient enrollment of an already funded clinical trial. Although we did not set out to systematically compare different strategies for enhanced enrollment, the narrative provided here introduces critical themes around enrollment barriers and remote delivery methods that are not unique to TSC. There is a high unmet need for treatment studies in rare neurodevelopmental disorders, yet challenges raised by the geographical distribution and the complex medical needs of these individuals often hamper effective recruitment and study success. The cost to participate in research, particularly intervention, is not only financial (lodging, airfare, meals) but also psychosocial, with disruption of routines and schedules, absence from home and work, and added caregiver burden. Study participation often requires caregivers to commit time during weekdays, requiring flexibility of employment hours, additional childcare, and availability of secondary caregivers. Other clinical trials for rare neurodevelopmental disorders have also begun to use remote intervention strategies, including the NeuroNext trial for Fragile $\mathrm{X}$ Syndrome (NCT02920892), which provides language intervention in conjunction with pharmacotherapy through a combination of clinic visits and at-home synchronous video conferencing sessions. Telehealth has additionally benefitted clinical care through models such as a hub and spoke network delivery system in which an anchor "hub" provides comprehensive expertise to secondary "spokes", including local healthcare providers [19]. One such model known as the Extension for Community Healthcare Outcomes (ECHO) has been applied to PhelanMcDermid Syndrome [20] and provides a platform for video-consultation between local physicians and academic experts to support clinical care in communities, thereby reducing the burden of care on both healthcare providers and caregivers. These telehealth models may prove effective in addressing challenges in recruitment for clinical trials, and importantly, disseminating academic expertise and evidence-based intervention to communities.

There are certainly limitations to remote delivery, including the potential for compromised standardization of protocols, reduced commitment to participation due to the lack of personal contact with study staff, and variable access to and comfort with technology for caregivers. More technological sophistication could further streamline and simplify the process for families, such as the use of a single cell phone application for data collection and intervention, or the replacement of all inperson visits with remote assessment tools. Varying doses of remote delivery need to be tested to determine the minimum amount of live interaction required to achieve measurable outcomes of interest.

Our remote adaptation of intervention directly addresses the barrier of distance, thus greatly increasing enrollment in the study from a geographically diverse pool of participants. Descriptively, the remote intervention cohort is also more representative than the small in-person cohort (a higher percentage of non-white and non-college educated caregivers); however, due to funding restrictions, the financial burden of travel was not fully addressed, and this barrier continues to limit the socioeconomic diversity of participants. In addition, there may be an inherent recruitment bias in the families that chose to engage in remote intervention, possibly 
selecting for families of infants that are more severely affected by comorbidities, lack financial resources, have limited access to clinically recommended early intervention programs, or live farther from TSC centers of excellence. One might argue that the subset of families who chose to enroll may not represent the broader TSC patient population due to selection factors, thus influencing the generalizability of this type of remote intervention delivery. To address this possible bias, we might consider the subgroup of families who were initially contacted for in-person intervention and then recontacted after modification. Of these families, $10 \%$ enrolled in the in-person intervention and $50 \%$ elected to participate when a remote option was made available, a lower proportion than the subsequent $82 \%$ that enrolled upon first contact. We did not collect demographic or clinical information on the infants that did not enroll, but this distinction does suggest inherent differences in cohorts based on study design and delivery methods that need to be considered when clinical outcomes are determined.

This study represents an important effort to increase access to intervention clinical trials, which will continue to remain the fundamental obstacle in treatment of rare neurodevelopmental disorders. The paradigm must shift from considering the "gold standard" as research conducted in academic centers to studies that promote family-centered, home-based delivery that maximize participation of all affected children. We also must emphasize that the data presented here are descriptive and meant to generate discussion around the need for innovation in trial design and delivery to maximize access and participation in rare neurodevelopmental disorders. From this experience, we may not be able to conclude that this particular remote delivery strategy is more effective for recruitment than alternative methods, such as the provision of in-home sessions with interventionists, web-based information delivery, or direct recruitment and intervention delivery that coincide with clinic appointments. A critical future direction will be to directly compare enrollment achieved through these various strategies.

\section{Conclusion}

Modification of this clinical trial to include remote delivery of intervention enhanced enrollment tenfold within 1 year and greatly improved the geographic and socioeconomic reach of the study. The striking, rapid surge in enrollment with these adjustments reinforces the tremendous motivation of these families to participate in clinical trials, as well as the need for behavioral intervention and rigorous testing of new approaches to enhance access to research in rare neurodevelopmental disorders. This telehealth model can facilitate future studies not only in rare conditions, but also in other populations that lack adequate access, such as families with limited financial or clinical resources. This study represents an important step towards building an evidence base for remote strategies to promote scalable access to intervention for children with rare genetic disorders, and it reveals a critical direction for future research inquiry as we identify which delivery methods are most effective for diverse populations.

\section{Abbreviations}

ASD: Autism spectrum disorder; ECHO: Extension for Community Healthcare Outcomes; IRB: Institutional Review Board; JASPER: Joint Attention, Symbolic Play, Engagement and Regulation; JETS: JASPER Early Intervention for Tuberous Sclerosis; TSC: Tuberous sclerosis complex

\section{Acknowledgements}

Not applicable.

\section{Authors' contributions}

$\mathrm{CH}$ collected and analyzed recruitment data and implemented methods adaptations and was a major contributor in writing the manuscript. MP, NM, and SJ were major contributors in writing the manuscript. All authors read and approved the final manuscript.

\section{Funding}

This study is funded by an R01 award from NIH, NCT03422367.

\section{Availability of data and materials}

The datasets generated and/or analyzed during the current study will be available in the NDAR repository upon completion of study.

\section{Ethics approval and consent to participate}

Families who enrolled in the study signed informed consent for usage and storage of de-identified data. This study was approved by the UCLA Medical IRB 3 committee, Protocol ID \#17-000262.

\section{Consent for publication}

Not applicable

\section{Competing interests}

The authors declare that they have no competing interests.

\section{Author details}

'UCLA Semel Institute for Neuroscience and Human Behavior, Los Angeles, CA 90095, USA. ${ }^{2}$ UCLA Graduate School of Education and Information Studies, Los Angeles, CA 90095, USA. ${ }^{3}$ UCLA Semel Institute, Los Angeles, CA 90095, USA. ${ }^{4}$ Boston Children's Hospital and Harvard Medical School, Harvard Graduate School of Education, Boston, MA 02115, USA. ${ }^{5}$ Massachusetts General Hospital and Harvard Medical School, Herscot Center for Tuberous Sclerosis Complex, Boston, MA 02114, USA.

Received: 6 September 2019 Accepted: 13 December 2019 Published online: 22 January 2020

\section{References}

1. Geschwind DH. Genetics of autism spectrum disorders. Trends Cogn Sci. 2011;15(9):409-16. https://doi.org/10.1016/j.tics.2011.07.003.

2. de la Paz MP, Villaverde-Hueso A, Alonso V, János S, Zurriaga O, Pollán M, Abaitua-Borda I. Rare diseases epidemiology research. Adv Exp Med Biol. 2010;686:17-39. https://doi.org/10.1007/978-90-481-9485-8_2.

3. Davis SA, Feldman SR. Using Hawthorne Effects to Improve Adherence in Clinical Practice: Lessons From Clinical Trials. JAMA Dermatol. 2013;149(4): 490-1. https://doi.org/10.1001/jamadermatol.2013.2843.

4. Carr T, Lord C. A pilot study promoting participation of families with limited resources in early autism intervention. Res Autism Spectr Disord. 2016;25: 87-96. https://doi.org/10.1016/j.rasd.2016.02.003.

5. Lord C, Bishop SL. Autism spectrum disorders: diagnosis, prevalence, and services for children and families. Soc Policy Rep. 2010;24(2) Retrieved from 
https://www.researchgate.net/publication/234582175_Autism_Spectrum_ Disorders_Diagnosis_Prevalence_and_Services_for_Children_and_Families_ Social_Policy_Report_Volume_24_Number_2.

6. Tek S, Landa RJ. Differences in autism symptoms between minority and non-minority toddlers. J Autism Dev Disord. 2012;42(9):1967-73. https://doi. org/10.1007/s10803-012-1445-8.

7. Ebrahimi-Fakhari D, Mann LL, Poryo M. Incidence of tuberous sclerosis and age at first diagnosis: new data and emerging trends from a national, prospective surveillance study. Orphanet J Rare Dis. 2018;13:117. https://doi. org/10.1186/s13023-018-0870-y.

8. Baio J, Wiggins L, Christensen DL, et al. Prevalence of Autism Spectrum Disorder Among Children Aged 8 Years - Autism and Developmental Disabilities Monitoring Network, 11 Sites, United States. MMWR Surveill Summ 2018. 2014;67(SS-6):1-23. https://doi.org/10.15585/mmwr.ss6706a1.

9. Asano E, Chugani DC, Muzik O, Behen M, Janisse J, Rothermel R, et al. Autism in tuberous sclerosis complex is related to both cortical and subcortical dysfunction. Neurology. 2001;57(7):1269-77. https://doi.org/10. 1212/wnl.57.7.1269.

10. Jeste SS, Varcin KJ, Hellemann GS, Gulsrud AC, Bhatt R, Kasari C, et al. Symptom profiles of autism spectrum disorder in tuberous sclerosis complex. Neurology. 2016;87(8):766-72. https://doi.org/10.1212/WNL. 0000000000003002 .

11. Datta AN, Hahn CD, Sahin M. Clinical presentation and diagnosis of tuberous sclerosis complex in infancy. J Child Neurol. 2008;23(3). https://doi. org/10.1177/0883073807309250.

12. Wortmann SB, Reimer A, Creemers JWT, Mullaart RA. Prenatal diagnosis of cerebral lesions in Tuberous sclerosis complex (TSC). Case report and review of the literature. Eur J Paediatr Neurol. 2008;12(2):123-6.

13. Jeste SS, Wu JY, Senturk D, Varcin K, Ko J, McCarthy B, et al. Early developmental trajectories associated with ASD in infants with tuberous sclerosis complex. Neurology. 2014;83(2):160-8. https://doi.org/10.1212/WNL. 0000000000000568 .

14. McDonald NM, Murphy HG, Messinger DS. Empathic responding in preschool-aged children with familial risk for autism. Autism Res. 2017; 10(10):1621-8. https://doi.org/10.1002/aur.1819.

15. Kasari C, Gulsrud A, Paparella T, Hellemann G, Berry K. Randomized comparative efficacy study of parent-mediated interventions for toddlers with autism. J Consult Clin Psychol. 2015;83(3):554-63. https://doi.org/10. 1037/a0039080.

16. Kasari C, Gulsrud AC, Wong C, Kwon S, Locke J. Randomized controlled caregiver mediated joint engagement intervention for toddlers with autism. J Autism Dev Disord. 2010;40(9):1045-56. https://doi.org/10.1007/s10803010-0955-5.

17. Kasari C, Lawton K, Shin W, Barker TV, Landa R, Lord C, et al. CaregiverMediated Intervention for Low-Resourced Preschoolers With Autism: An RCT. Pediatrics. 2014;134(1):e72. https://doi.org/10.1542/peds.2013-3229.

18. Shire SY, Chang YC, Shih W, Bracaglia S, Kodjoe M, Kasari C. Hybrid implementation model of community-partnered early intervention for toddlers with autism: a randomized trial. J Child Psychol Psychiatry. 2016; 58(5):612-22. https://doi.org/10.1111/jcpp.12672.

19. Elrod JK, Fortenberry JL Jr. The hub-and-spoke organization design: an avenue for serving patients well. BMC Health Serv Res. 2017;17(Suppl 1):457. https://doi.org/10.1186/s12913-017-2341-x.

20. Zhou C, Crawford A, Serhal E, Kurdyak P, Sockalingam S. The impact of project ECHO on participant and patient outcomes: a systematic review. Acad Med. 2016;91(10):1439-61. https://doi.org/10.1097/ACM. 0000000000001328.

\section{Publisher's Note}

Springer Nature remains neutral with regard to jurisdictional claims in published maps and institutional affiliations.

Ready to submit your research? Choose BMC and benefit from:

- fast, convenient online submission

- thorough peer review by experienced researchers in your field

- rapid publication on acceptance

- support for research data, including large and complex data types

- gold Open Access which fosters wider collaboration and increased citations

- maximum visibility for your research: over $100 \mathrm{M}$ website views per year

At BMC, research is always in progress.

Learn more biomedcentral.com/submissions 